\title{
Diet quality, usage and perceived benefits of nutritional supplements in young healthy adults
}

\author{
S.A. Lowry, I. Alaunyte and F. Amirabdollahian \\ School of Health Sciences, Liverpool Hope University, Liverpool, L16 9JD
}

Adequate nutritional status can be obtained by following a balanced diet. Hence the use of dietary supplements in populations not at risk of nutrient deficiencies is not recommended ${ }^{(1)}$. Nonetheless the dietary supplement industry is ever expanding and estimated to be worth around $£ 385$ million in the $\mathrm{UK}^{(2)}$. There is a growing concern that the increase in supplementation of the diet directly increases the incidence of improper use and accidental overdose of these nutrients ${ }^{(3)}$. The aim of this study was to determine whether the usage of supplements provide nutritional adequacy in young healthy adults and to explore the reasons for taking supplements.

After obtaining ethical approval, participants ( $n$ 238, M 104, F 134) aged 18-25 from universities in the North West region were recruited via convenient and snowball sampling. Frequency, quantity and reasons of supplement consumption were assessed using a validated questionnaire ${ }^{(3)}$. Nutrient intake was assessed by a validated 3 day food diary and analysed using dietary assessment software Microdiet. Normal distribution was investigated using the Shapiro-Wilk test of normality. Statistical analyses comparing supplement users to none users were conducted using SPSS 22. Questionnaires were analysed using chi-squared test and food diaries analysed using an independent t-test. Statistical significance was set at 0.05.

Findings revealed that $48 \%$ of participants reported current use of dietary supplements. This was significantly higher in males compared to females $(65 \% \vee 35 \%$, respectively, $\mathrm{P}<0.05)$. The main reasons for supplement use were safety of supplements, followed by provision of more energy, improved training, endurance and health outcomes. Supplement users had significantly higher intakes of Vitamin C, Magnesium and Zinc. Significantly more energy came from carbohydrates in non-users whilst significantly more energy derived from protein in supplement users.

\begin{tabular}{|c|c|c|c|c|c|}
\hline \multirow[t]{2}{*}{ Nutrient } & \multicolumn{2}{|c|}{$\begin{array}{l}\text { Supplement } \\
\text { users }(n 115)\end{array}$} & \multicolumn{2}{|c|}{$\begin{array}{c}\text { Non-users } \\
(n \text { 123) }\end{array}$} & \multirow[t]{2}{*}{$\begin{array}{c}P \\
\text { value }\end{array}$} \\
\hline & $\begin{array}{c}\text { Mea } \\
\mathbf{n}\end{array}$ & SD & $\begin{array}{c}\text { Mea } \\
\mathbf{n}\end{array}$ & SD & \\
\hline $\begin{array}{l}\text { Carbohydrate } \\
(\% \text { TEI) }\end{array}$ & 42.0 & 8.4 & 46.0 & 9.1 & 0.001 \\
\hline $\begin{array}{l}\text { Protein }(\% \\
\text { TEI) }\end{array}$ & 21.0 & 8.1 & 18.0 & 6.8 & 0.001 \\
\hline Fat (\% TEI) & 34.0 & 7.0 & 34.0 & 7.6 & 0.901 \\
\hline $\begin{array}{l}\text { Vitamin D } \\
(\mu \mathrm{g} / \text { day })\end{array}$ & 12.3 & 46.1 & 5.6 & 10.7 & 0.134 \\
\hline $\begin{array}{l}\text { Vitamin C } \\
\text { (mg/day) }\end{array}$ & 123.0 & 214.9 & 81.0 & 70.8 & 0.046 \\
\hline $\begin{array}{l}\text { Vitamin B12 } \\
\text { (mg/day) }\end{array}$ & 6.0 & 12.0 & 4.1 & 8.2 & 0.164 \\
\hline $\begin{array}{l}\text { Retinol } \\
\text { (mg/day) }\end{array}$ & 348.1 & 635.5 & 270.3 & $\begin{array}{l}354 . \\
4\end{array}$ & 0.240 \\
\hline $\begin{array}{l}\text { Calcium } \\
\text { (mg/day) }\end{array}$ & 722.8 & 321.0 & 718.4 & $\begin{array}{l}436 . \\
0\end{array}$ & 0.930 \\
\hline Iron (mg/day) & 17.7 & 46.8 & 10.1 & 5.1 & 0.840 \\
\hline $\begin{array}{l}\text { Magnesium } \\
\text { (mg/day) }\end{array}$ & 277.3 & 120.8 & 239.0 & 96.7 & 0.007 \\
\hline $\begin{array}{l}\text { Zinc } \\
\text { (mg/day) }\end{array}$ & 9.9 & 5.3 & 8.0 & 3.7 & 0.002 \\
\hline
\end{tabular}

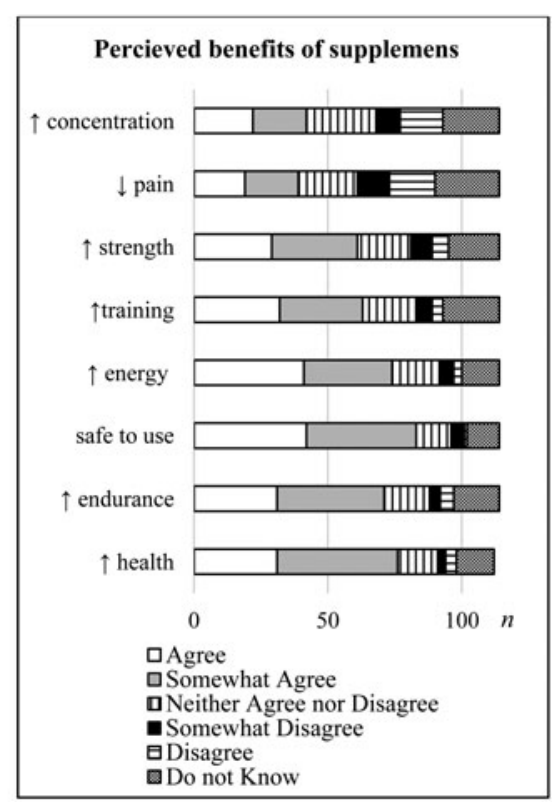

In conclusion, similarly to previous research ${ }^{(4)}$, individuals who consume dietary supplements appear to achieve higher intakes of micronutrients. However the proportions of energy derived from macronutrients was better balanced in non-users.

1. Kadetz (2015) J Ethnopharmacol [Epub ahead of print].

2. Houser, Kennedy and Luo (2013) Ecol Food Nutr 52, 76-84.

3. Aljaloud and Ibrahim (2013) J Nutr Metab 2013, 1-7.

4. Wiltgren, Booth, Kaur et al. (2015) Nutr 7, 1094-1107. 